\title{
Patients' Complaints after Scaling and the Self-evaluation of Hand Instrumentation for Scales by Dental Hygienists
}

\author{
Jo Se $\operatorname{Rim}^{1}$ and Hee Ja $\mathrm{Na}^{2 *}$ \\ ${ }^{1}$ Department of Dental Hygiene, Dankook University, Korea \\ ${ }^{2}$ Department of Dental Hygiene, Honan University, Korea \\ *Corresponding author: Hee Ja Na, Department of Dental Hygiene, Honan University, Kwangju, Korea
}

\begin{abstract}
Objectives: This study was performed to understand such chief complaints through scaling operation performed by dental hygienists, as bleeding, or instrument sensation for dental patients and how to improve by explain the control method of them, in order to supply the better operation in the future and contribute the improve for the patient's oral health.

Subjects and Method: Thirty five female dental patients who had received the scaling from 35 dental hygienists were participated in this clinical study, from the periods on March, in the year 2020 at G city, and the questionnaire method was performed with such questions as chief complaints after scaling with Likert 5 scale scores.

Results: The complaints or merits after scaling were examined as followings as bleeding complaint was $3.51 \pm 1.29$ points, hyper-sensation of the tooth was $3.74 \pm 1.26$ points, tooth crack or fracture as $2.68 \pm 1.18$ points, abrasion as $4.02 \pm 1.15$ points, possible for eating hot and hard foods as $3.20 \pm 1.32$ points, early detect of caries as $2.17 \pm 0.92$, points, chance for consultation about implant or denture as $3.22 \pm 1.19$, possible for drinking acidic beverage as $3.20 \pm 1.32$,points , possible for finding enamel hypoplasia as $2.20 \pm 1.23$, points and possible for removal of filling or prosthodontic materials as $2.88 \pm 1.30$ points. The evaluation of dental hygienist for the hand instrument manage skill is revealed as $4.11 \pm 1.10$ points for supra-gingival calculus removal, $2.51 \pm 1.29$ points for sub-gingival calculus removal, $4.17 \pm 0.95$ points for using $1 \sim 2 \mathrm{~mm}$ lateral surface of scaler blade, $2.77 \pm 1.23$ points for using the back surface of the scaler blade, $2.60 \pm 1.11$ points for start scaling after demonstration with the tip guide. $3.22 \pm 1.35$ points for grasping scaler slightly and insert it around the tooth as like as probe use, $3.14 \pm 1.37$ points for vertical movement with slow motion of the tip along the long axis of the tooth on sub-gingival area, $2.88 \pm 1.34$ points for using scaler tip with slight pumping touch at the tooth surface, $3.48 \pm 1.33$ points for moving the blade vertically with inserting it on col area and $2.94 \pm 1.34$ points for put the scaler tip locate beside the proximal area of the neighbor tooth on scaling at proximal area.
\end{abstract}

Conclusion: The abrasion or hypersensitivity were the big complaints from patients on scaling and dental hygienists were evaluated as high score in such item of scaling skills as supra-gingival calculus removal and using $1 \sim 2 \mathrm{~mm}$ on lateral surface of scaler blade, but got the least score in such item on sub-gingival calculus removal.

Keywords: Dental Scaling; control dental; dental hygienist; pain; dental calculus

\section{Introduction}

The periodontal care can be performed through such method as the non-operative periodontal treatment, operation and scaling or oral prophylaxis and professional mechanical tooth cleaning. Non-surgical periodontal care is aims for diminishing the gingival inflammation and eliminate the periodontal pocket through the removal of dental plaque and calculus surrounding the tooth, in order to re-attach or re-generation of the periodontal tissue.
Moreover, it can be done as a pre-treatment procedure for severe periodontal surgery through performing the removal of plaque and calculus, polishing the root surface, applying the chemical therapy or occlusal re-adjustment. On the other hands, surgical periodontal treatment can be determined as operation surrounding gingival tissue through incision or excision of the periodontal tissue. It can be operated the calculus removal and root planning through the security of operating sight and re-forming of the gingival out- 
line form through the suture after operation, in order to easy success for oral hygiene [1]. Scaling can be defined as the removal of plaque and deposit calculus on the tooth surface as well as the removal of tooth stain as caffeine or nicotine, in order to diminish the micro-organisms or changing the compositions at sub-gingival area, to result for prevention of periodontal disease by eliminating the etiology of tooth loss, as an effective method for periodontal disease. Periodontal disease is one of the most popular dental disease in the world-wide and dental hygienist is one of the proper dental workforces to manage the periodontal disease through performing the preventive cares as scaling. it should be needed for expert skill for scaling for dental hygienist and one pf the skill check would be instrumentation skill check for them at each potion of detailed areas at oral portions, because of elimination or diminish the oral micro-organisms through the removal of calculus or plaque by expert scaling. Although the scaling is a good measure to prevent periodontal disease, there has been revealed such complaints from the patients as hypersensitivity of the tooth, gingival bleeding, pain, abrasion of the cervical area of the tooth, removal of the filling or prosthodontic materials and the operators have to find their chief complaints to notice them to patients or do their best to avoid it with careful skills on scaling. It would be a good way for operator as dental hygienist to learn and training the bleeding control or stopping method before scaling about the procedure and anatomical structure of the blood vessel in the oral cavity or blood pressure control method or drug of choice as using Epinephrine derivatives or Bosmin agent. Another complaints or side effect from the scaling should be understood for operators in order to diminish them to perform the successful scaling. The aims of this study have been suggested to understand the chief complaint or oral status changes as well as checking and evaluation the operators scaling skill done by dental hygienist, in order to supply more safe and expert operation to dental patient in the future.

\section{Subjects and Method}

\section{Subjects}

Thirty five dental hygienists who have been working at dental clinics at Gwangju city, Korea were participated in this study for checking the scaling skill and also 35 dental patients who has visited dental clinic and received the scaling by 35 dental hygienists were joined to ask for chief complaint or side effect after scaling.

\section{Method}

This questionnaire method of examination was performed after achieved the certification for IRB (Institutional Review Board) from Bio ethic committee of Honam University as number as 1041223 201912-HR 18. Self-evaluation for managing the hand instrumentation skill of scaling by-themselves with using the 5 scaled score evaluation sheet after scaling, at Kwangju city on March in the year 2020. 10 questions such as supra-gingival calculus removal, sub-gingival calculus removal, using 1 2 mm lateral surface of scaler blade, using the back surface of the scaler blade, adjusting tip guide for scaling. grasping scaler slightly and insert it around the tooth as like as probe use, vertical movement with slow motion of the tip along the long axis of the tooth on subgingival area, using scaler tip with slight pumping touch at the tooth surface, moving the blade vertically with inserting it on the cervical area and putting the scaler tip insertion with proper location at the proximal area of the neighbor tooth on scaling at proximal area. Also 35 of their patients for scaling were joined to be asked for some complaints or side effects of scaling with use of 9 questions such as bleeding complaint, hyper-sensation of the tooth,, tooth crack or fracture, abrasion, possible for eating hot and hard foods, early detect of caries, chance for consultation about implant or denture, possible for drinking acidic beverage, possible for finding enamel and possible for removal of filling or prosthodontic materials, by supplying the questionnaire sheet. The data was collected through the calculation of average mean points and standard deviation with 5 points as full score for each question and compared and analysis with their points with statistical analysis. Data were analyzed by use of the computer program as SPSS 18.0 for the data of mean and standard deviation with $t$ value and significance, for 5 points of full score for each question.

\section{Result}

\section{General characteristics for subjects}

(Table 1). Through the general characteristics for patients of this study, the oral hygiene level was $3.00 \pm 0.72$ points, gingival bleeding was $1.20 \pm 0.40$ points, tooth-brushing level was $2.37 \pm$ 0.49 points and the frequency of scaling was examined as $1.22 \pm 0.42$ points. The average age of the subjects was $45.25 \pm 9.45$ years old (Table 2). The complaints or merits after scaling were examined as followings as bleeding complaint was $3.51 \pm 1.29$ points, hypersensation of the tooth was $3.74 \pm 1.26$ points, tooth crack or fracture as $2.68 \pm 1.18$ points, abrasion as $4.02 \pm 1.15$ points, possible for eating hot and hard foods as $3.20 \pm 1.32$ points, early detect of caries as $2.17 \pm 0.92$, points, chance for consultation about implant or denture as $3.22 \pm 1.19$, impossible for drinking acidic beverage as $2.80 \pm 1.32$, points, possible for finding enamel hypoplasia as $2.20 \pm 1.23$, points and possible for removal of filling or prosthodontic materials as $2.88 \pm 1.30$ points (Table 3 ). The evaluation of dental hygienist for the hand instrument manage skill is revealed as $4.11 \pm 1.10$ points for supra-gingival calculus removal, $2.51 \pm 1.29$ points for sub-gingival calculus removal, $4.17 \pm 0.95$ points for using 1 2 mm lateral surface of scaler blade, $2.77 \pm 1.23$ points for using the back surface of the scaler blade, $2.60 \pm 1.11$ points for adjusting tip guide for scaling. $3.22 \pm 1.35$ points for grasping scaler slightly and insert it around the tooth as like as probe use, $3.14 \pm 1.37$ points for vertical movement with slow motion of the tip along the long axis of the tooth on sub-gingival area, $2.88 \pm 1.34$ points for using 
scaler tip with slight pumping touch at the tooth surface, $3.48 \pm 1.33$ with proper location at the proximal area of the neighbor tooth on points for moving the blade vertically with inserting it on the scaling at proximal area.

cervical area and $2.94 \pm 1.34$ points for put the scaler tip insertion

Table 1: General characteristics for patient subjects ( $n=35$, full score $=5$.0points).

\begin{tabular}{|c|c|c|c|c|c|}
\hline Item & Sub Item & n & $\%$ & Mean & SD \\
\hline Oral Hygiene & Well-done & 9 & 25.7 & \multirow{3}{*}{3} & \multirow{3}{*}{0.72} \\
\hline \multirow{2}{*}{ Care } & Poor & 17 & 48.6 & & \\
\hline & Very Poor & 9 & 25.7 & & \\
\hline \multirow{2}{*}{ Bleeding } & Yes & 28 & 80 & \multirow{2}{*}{1.2} & \multirow{2}{*}{0.4} \\
\hline & No & 7 & 20 & & \\
\hline \multirow{2}{*}{ Tooth-Brushing } & 2 Times/day & 22 & 62.9 & \multirow{2}{*}{2.37} & \multirow{2}{*}{0.49} \\
\hline & 3 times/day & 13 & 37.1 & & \\
\hline \multirow{2}{*}{ Scaling } & 1 time/Year & 27 & 77.1 & \multirow{2}{*}{1.22} & \multirow{2}{*}{0.42} \\
\hline & 2 Times/Year & 8 & 22.9 & & \\
\hline \multirow{5}{*}{ Age } & Average & 35 & & \multirow{5}{*}{45.25} & \multirow{5}{*}{9.45} \\
\hline & $30 \mathrm{~S}$ & 14 & 40 & & \\
\hline & $40 \mathrm{~S}$ & 4 & 11.6 & & \\
\hline & $50 \mathrm{~S}$ & 14 & 45 & & \\
\hline & Over 60 & 1 & 2.9 & & \\
\hline
\end{tabular}

Table 2: Patient's complaint the oral and habitual state changes after scaling. (Full score=5.0 points, $\mathrm{n}=35)$.

\begin{tabular}{|c|c|c|c|c|c|}
\hline Item & Sub-Item & Mean & SD & t & p \\
\hline Patient complaint & Gingival Bleeding & 3.51 & 1.29 & 1.08 & 1.89 \\
\hline \multirow{5}{*}{$\begin{array}{c}\text { Oral Status or } \\
\text { Symptom }\end{array}$} & Hypersensitive tooth & 3.74 & 1.26 & 13.43 & 0.32 \\
\cline { 2 - 6 } & Tooth crack/fracture & 2.68 & 1.18 & 20.72 & 0 \\
\cline { 2 - 6 } & Tooth abrasion & 4.02 & 1.15 & 14.29 & 0 \\
\cline { 2 - 6 } & Impo.-Hot \& hard food & 3.2 & 1.32 & 13.91 & 0 \\
\cline { 2 - 6 } & Early caries detects & 2.17 & 0.92 & 16.04 & 0 \\
\cline { 2 - 6 } & Implant denture & 3.22 & 1.19 & 12.51 & 0 \\
\cline { 2 - 6 } & Impo. Acidic beverage & 2.8 & 1.32 & 10.56 & 0 \\
\cline { 2 - 6 } & Enamel hypoplasia & 2.2 & 1.3 & 13.12 & 0 \\
\cline { 2 - 6 } & Removal of fill/prosth. & 2.88 & & & 0 \\
\hline
\end{tabular}

Impo.: Impossible for eating, Impo. Acidic beverage; Impossible for drinking acidic beverage, Prostho: Prosthodontic appliance material.

Table 3: Self-evaluation points for the skill of the hand instrumentation for scaling done by dental hygienist.

\begin{tabular}{|c|c|c|c|c|}
\hline Item & Mean & S.D & t & p \\
\hline supra-gingival calculus removal & 4.11 & 1.1 & 22.02 & 0 \\
\hline sub-gingival calculus removal & 2.51 & 1.29 & 25.85 & 0 \\
\hline using 1 2 mm lateral blade & 4.17 & 0.95 & 13.23 & 0 \\
\hline Using back side tip scaler blade & 2.77 & 1.23 & 13.77 & 0 \\
\hline adjusting tip guide for scaling & 2.6 & 1.11 & 14.12 & 0 \\
\hline insert scaler with slight grasp & 3.22 & 1.35 & 13.52 & 0 \\
\hline slow down motion at sub-gingiva & 3.14 & 1.37 & 12.68 & 0 \\
\hline using tip as slight pumping touch & 2.88 & 1.34 & 15.42 & 0 \\
\hline vertical movement at the col area & 3.48 & 1.33 & 12.9 & \\
\hline proper tip inserts at proximal area & 2.94 & 1.34 & & 0 \\
\hline
\end{tabular}

$(\mathrm{n}=35$, full score $=5.0$ points $)$. 


\section{Discussion}

Scaling and root planning are very important for dental treatments. Scaling is a process eliminating plaque, calculus, necrotic tissue and so on, and root planning makes root surfaces of infected teeth smooth after scaling. Scaling and root planning reduce the number of sub-gingival pathogenic organisms, and they return unhealthy sub-gingival to a healthy state; therefore, they promote healing of periodontal tissue by inducing epithelial tissue attached to root surface. Until now, the studies about the effectiveness of hand and powered instruments for scaling and root planning, the influence on changes in the root surface and dentin hypersensitivity after periodontal treatment have been conducted. The differences of studies about the effectiveness of various instruments for scaling and root planning and the influence on changes in the root surface were shown by a type of instrument, sharpness, the experimental method of in vivo and in vitro, time and the frequency of strokes, pressure on the instruments and the contact angles. Table 1 showed the general characteristics of patients of subjects and the oral hygiene state was $3.00 \pm 0.72$ points in 5.0 points of full score as appraised as normal state of oral hygiene. It was distributed as 9 as $25.7 \%$ in well state, 17 persons as $48.6 \%$ in poor and the rest 9 as $25.7 \%$ in severe poor state. Gingival bleeding score was checked as $1.20 \pm 0.40$ points as much subjects were found as bleeding. $80.0 \%$ of them have bleeding as usual and 7 persons as $20.0 \%$ of them had no bleeding at all before scaling. Tooth-brushing level was checked as $2.37 \pm 0.49$ points estimated as general level and the frequency of scaling level was as $1.22 \pm 0.42$ points as low level. The average age of them was $45.25 \pm 9.45$ years old as middle-aged group. This study was performed for checking patient's complaint for scaling or some symptoms or side effect from receiving the scaling, and these data from the patients would be contribute to understand them and to minimize the complaint or side effect for scaling by dental hygienists in the future. Recently, a various oral application method has been studied like varnish using mucus which is mixed $5 \% \mathrm{NaF}$ with colophony resin [2].

Age and oral health consciousness affect its importance [3] oral health disorder Ju et al. [4] reported that the coverage of insurance coverage twice a year and the expansion from the high school level would be more appropriate than the current one. Oral health beliefs about the level of beneficial effects of toothbrush behavior were higher for subjects who wore toothbrushes three times a day and wore toothbrushes before going to bed [5]. Recently, it has been recognized that the symbiotic status of bacteria in the oral cavity is important because bacterial diversity is maintained, rather than antibacterial concept which reduces microorganisms themselves [6]. Table 2 showed that the complaints or merits after scaling were examined as followings as bleeding complaint was $3.51 \pm 1.29$ points, hyper-sensation of the tooth was $3.74 \pm 1.26$ points, tooth crack or fracture as $2.68 \pm 1.18$ points, abrasion as $4.02 \pm 1.15$ points, possible for eating hot and hard foods as $3.20 \pm 1.32$ points, early detect of caries as $2.17 \pm 0.92$, points, chance for consultation about implant or denture as $3.22 \pm 1.19$, impossible for drinking acidic beverage as $2.80 \pm 1.32$,points , possible for finding enamel hypoplasia as $2.20 \pm 1.23$, points and possible for removal of filling or prosthodontic materials as $2.88 \pm 1.30$ points. It means that the important chief complaints of scaling patients were gingival bleeding and hypersensitivity after scaling. It could be occurred the gingival bleeding through irritating the inflammatory gingiva on the time of the removal of calculus and hypersensitive dentin could be occurred by removal of calculus which would be a role of protection from outer irritation as cold or hot air or water, at the cervical area of the tooth surface. it needed to let patient understands this theory of bleeding and hypersensitivity through the oral education before scaling. The other symptoms were revealed as better after scaling than before, at large or a little bit. Anyway, patient education to inform the side effect or symptoms should be done to dental patient for scaling before operation. Park et al. [7] emphasize that the oral health belief on level of beneficial impact according to the toothbrush behavior was higher for the subjects with toothbrush 3 times a day and toothbrush before going to bed.

The evaluation of dental hygienist for the hand instrument manage skill is revealed as $4.11 \pm 1.10$ points for supra-gingival calculus removal, $2.51 \pm 1.29$ points for sub-gingival calculus removal, $4.17 \pm 0.95$ points for using $1 \sim 2 \mathrm{~mm}$ lateral surface of scaler blade, $2.77 \pm 1.23$ points for using the back surface of the scaler blade, $2.60 \pm 1.11$ points for adjusting tip guide for scaling. $3.22 \pm 1.35$ points for grasping scaler slightly and insert it around the tooth as like as probe use, $3.14 \pm 1.37$ points for vertical movement with slow motion of the tip along the long axis of the tooth on sub-gingival area, $2.88 \pm 1.34$ points for using scaler tip with slight pumping touch at the tooth surface, $3.48 \pm 1.33$ points for moving the blade vertically with inserting it on the cervical area and $2.94 \pm 1.34$ points for put the scaler tip insertion with proper location at the proximal area of the neighbor tooth on scaling at proximal area. From the results, dental hygienists could well perform the elimination of the calculus at the supra gingival deposition otherwise hard for sub-gingival portion. it should be training more and re-examination system for remnant deposition of calculus would be recommended to educate on training cause. Also, it revealed high score for using side surface of the scaler blade but low score to use a back side of scaler blade on scaling. It revealed average 2.5 points to 3.5 points for all check items. It means that the most dental hygienists could perform the scaling with middle leveled or a little bit high leveled ability and left some upgrade in the future. it would be recommended to training repeatedly for dental hygienists to operate scaling with one step by one step periodically.

\section{Conclusion}

This study was performed with questionnaire method about the patient's complaint for scaling and self -evaluation for hand instrumentation skill done by dental hygienist in order to deliver 
the operation with improvement in the future. 35 dental hygienists who have been working at dental clinics in Kwangju city and 35 their scaling patients were participated in this study. The questionnaires for complaints or side effects were checked for scaling patients and hand instrumentation skills were checked for self-evaluation for dental hygienists after scaling. From the results, the obtained conclusion was as followings.

a) It was revealed that the main complaints after scaling were gingival bleeding and hypersensitive dentin and the others were not so big leveled problems.

b) Dental education for patient's complaints or side effects should be done before scaling operation in order to minimize the patient's complaints.

c) Dental hygienists have done well for removal of supragingiva calculus deposit and using 1 2 $\mathrm{mm}$ lateral surface of scaler blade, but not so high leveled score in the other items, left to be improvement.

d) Periodic training for dental hygienist should be needed to remind and to improve the hand instrumentation skill for scaling.

\section{Acknowledgements}

I express my gratitude to the dentist and the dental hygienist Moon Sun-Hee who contributed to the writing of this paper. We also thank patients and dental hygienists who participated in the questionnaire as individuals who contributed research materials, expertise, or time.

\section{Conflict of Interest}

There is no economic interest or conflict of interest.

\section{References}

1. Dasanayake AP (2012) Scaling and Root Planing Performed on Pregnant Women with Mild to Moderate Periodontal Disease is Not Efficacious in Reducing Preterm Birth, Low Birth Weight, or Other Poor Pregnancy Outcomes. Journal of evidence based dental practice 12(3): 135-137.

2. Kim HJ, Lee CH, Park JH, Chung YB (2009) Fluoride uptake on the tooth enamel according to application method. Int J Clin Prev Dent 5(1): 39-47.

3. Hwang HR, Cho YS (2011) The effect of oral health belief influence on the preventive oral health behaviors in college students. Int Clin Prev Dent 7: 135-140.

4. Ju OJ, Kang EJ, Woo SH, Lee AJ, Lee HJ, et al. (2014) Recognition between laypersons and dental hygienists on expansion of health insurance of scaling. J Korean Soc Dent Hyg 14(3): 431-438.

5. Park JY, Shin SC, Park YD, Chon JA, Cho JW (2013) Relevance between self-rated oral health and oral health behaviors in Korean adolescents: 2010 Korea Center for Disease Control. Int J Clin Prev Dent 9: 99-104.

6. Lynge Pedersen AM, Belstrøm D (2019) The role of natural salivary defences in maintaining a healthy oral microbiota. J Dent 80 (Suppl 1): $3-12$.

7. Park JY, Shin SC, Park YD, Chon JA, Cho JW (2013) Relevance between self-rated oral health and oral health behaviors in Korean adolescents: 2010 Korea Center for Disease Control. Int J Clin Prev Dent 9: 99-104.
To Submit Your Article Click Here: This work is licensed under Creative Commons Attribution 4.0 License

Submit Article

DOI: $10.32474 /$ IPDOAJ.2020.04.000191

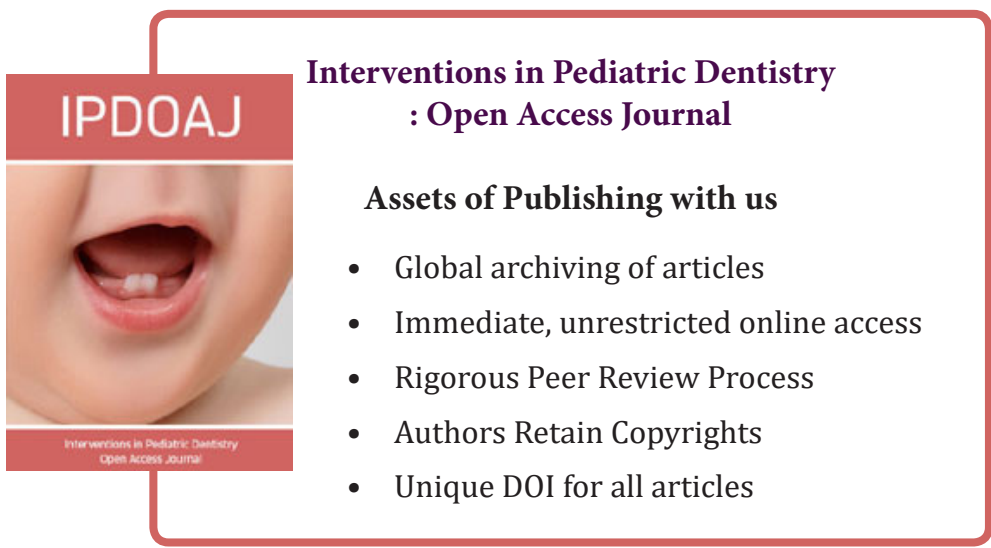

